\title{
4 La fine del mondo nella diade centrale
}

La diade centrale è senza dubbio la sezione del De rerum natura dove si riscontra una minore rilevanza del tema della fine del mondo. La principale ragione di tale situazione risiede nella tematica dei libri III e IV, incentrati rispettivamente sulla natura dell'anima e sulla percezione sensoriale, ovverosia sul "microcosmo" umano. Non mancano tuttavia alcuni passi dove è possibile individuare richiami alla tematica escatologica, che pare opportuno segnalare in questo breve capitolo.

\subsection{Il proemio "apocalittico" del terzo libro}

Il terzo libro del De rerum natura si apre con una visione cosmica che sembra celebrare il definitivo superamento degli stretti confini di questo mondo a favore di una prospettiva "universale" (vv. 14-18; 25-30):

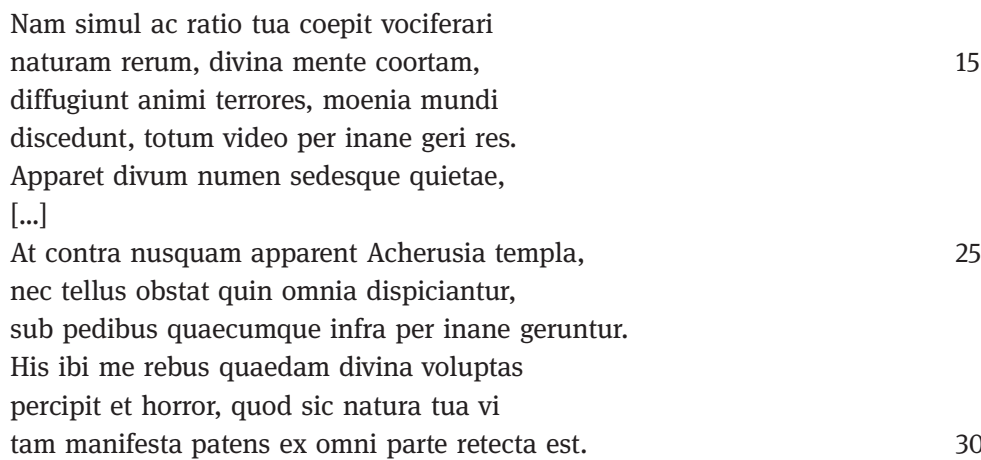

15 coorta Orelli : coortam $O$ : coartam QV 28 ibi Pontanus : ubi OQV 29 sic natura Avancius : signatura $O Q V$

Non appena infatti il tuo senno cominciò a proclamare

a gran voce la natura delle cose, sbocciata nella tua mente divina, dileguano i terrori dell'animo, le mura del mondo si infrangono e le cose io vedo prodursi nel vuoto infinito. Appare il nume degli dèi e le sedi quiete [...] Invece non appaiono in nessun luogo i templi acherontei né d'altra parte la terra impedisce che si scorga tutto ciò che sotto i nostri piedi si produce nello spazio vuoto. Davanti a tali cose un certo divino piacere 
e un brivido s'impadroniscono di me, poiché per tua forza

d'intelletto la natura si apre a noi, svelata in ogni sua parte.

La rivelazione di Epicuro ha ormai "scoperchiato" i moenia mundi, dischiudendo la visione degli dèi negli intermondi, dell'universo infinito e delle sue eterne componenti: gli atomi e il vuoto. Come si è visto nell'introduzione alla precedente sezione, l'espressione moenia mundi discedunt sembra celare un riferimento alla dottrina epicurea della mortalità del mondo: nella Gigantomachia rovesciata di 1.62-79 la vittoria del "ribelle" Epicuro si fondava proprio sullo scardinamento del cielo, che apriva le porte all'immenso spazio oltre il mondo. Solo riconoscendo la mortalità del mondo è possibile "conquistare" intellettualmente l'infinito e contemplare gli dèi negli intermondi, ${ }^{1}$ così da raggiungere infine la pace interiore. Questa non è però l'unica possibile allusione escatologica presente nel proemio del terzo libro. Nei vv. 25-27 Lucrezio descrive l'apertura dell'immensità e del vuoto sotto i piedi di colui che scopre la verità. Questa mistica visione pur prendendo le mosse dalle parole di Epicuro stesso, ${ }^{2}$ sembra al contempo una riformulazione di un motivo topico negli scenari “apocalittici” e dell'epica, ovverosia lo scoperchiamento dell'Ade e il disvelamento del mondo sotterraneo. ${ }^{3}$ Del resto, questa tipologia di scenario è annoverata tra i maggiori esempi di sublime nel trattato Sul sublime (9.6): «ti figuri, amico mio, la terra squarciata dalle sue fondamenta, il Tartaro messo a nudo, tutto l'universo rovesciato e sconvolto, e ogni cosa mescolata: cielo e Ade, cose mortali e immortali che si affrontano e lottano nella stessa battaglia?». ${ }^{4}$

Come sempre, la ripresa lucreziana implica una originale rielaborazione del motivo topico. Difatti, secondo il poeta, aprendosi sotto i piedi dell'umanità ("illuminata" da Epicuro), la terra non rivela più le case dell'Ade di cui parlano il mito e la religio (nusquam apparent Acherusia templa), bensì, come nel sublime finale del primo libro (vv. 1106 ss. terraque se pedibus raptim subducat), l'infinito universo. Dunque l'antico sublime escatologico viene riformulato per pervenire a una nuova tipologia di v̌ $\psi \varsigma$ s epicureo, che trascende il sublime della tradizione. Fonte di horror e di divina voluptas non sono più le scene di Teomachia e Gigantomachia, bensì la visione cosmica sub specie aeternitatis della natura

1 A proposito del motivo della contemplazione degli dèi negli intermondi si veda Segal 1998, 68-69 e l'Appendice 4.

2 Cfr. Epic. fr. 299 Usen. (= Plut. De defectu 28.425d) ed ep. Hdt. 60.

3 Cfr. e.g. Hom. Il. 20.61-65.

4 Per il testo greco di questo passo e un commento più ampio, cfr. pp. 261-266. 
dell'universo, permessa da Epicuro. Questo aspetto verrà approfondito nei capitoli finali della nostra ricerca, dedicati alla natura del sublime lucreziano. ${ }^{5}$

\subsection{Sublime escatologico ed epica patriottica (3.830-842)}

Ai vv. 847-853 Lucrezio spiega come sia possibile che, nel corso dell'eternità, i medesimi atomi abbiano dato vita a individui identici. ${ }^{6}$ Tuttavia, ciò non ha implicazione alcuna per la vita di ciascuno di tali individui, poiché la morte costituisce un limite invalicabile per la percezione e per la memoria della loro esistenza. Lo stesso ragionamento è implicitamente valido per ogni aggregato atomico, dalle città al mondo: non uno, ma infiniti mondi equivalenti al nostro potrebbero essere esistiti nell'eterno tempo dell'universo infinito; parimenti, innumerevoli città di Roma con il loro imperium potrebbero esistere o essere esistite in altri mondi nel passato e potrebbero nascere in futuro. La summa di queste conclusioni tende a minare alla base ogni ideologia fondata sulla fede nell'unicità, nella provvidenzialità e nell'eternità del cosmo, e, parimenti, dello stato romano. ${ }^{7}$

Il sarcasmo lucreziano nei confronti dell'ideologia della città eterna e sovrana del mondo è del resto evidente nei versi che precedono immediatamente quelli appena citati (vv. 830-842):

Nil igitur mors est ad nos neque pertinet hilum, 830 quandoquidem natura animi mortalis habetur. Et velut anteacto nil tempore sensimus aegri, ad confligendum venientibus undique Poenis, omnia cum belli trepido concussa tumultu horrida contremuere sub altis aetheris oris, 835 in dubioque fuere utrorum ad regna cadendum omnibus humanis esset terraque marique, sic, ubi non erimus, cum corporis atque animai discidium fuerit quibus e sumus uniter apti, scilicet haud nobis quicquam, qui non erimus tum, 840 accidere omnino poterit sensumque movere, non si terra mari miscebitur et mare caelo.

835 oris Gifanius : auris $O$ : auras $Q$

5 Cfr. pp. 252-270.

6 Cfr. il commento di Schiesaro 1996, 795-804, che dimostra in maniera persuasiva che qui

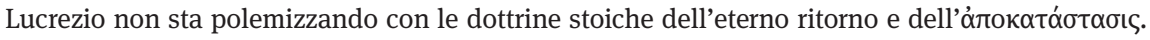
7 Cfr. Schiesaro 2007a. 
Nulla è dunque la morte per noi, e per niente ci riguarda,

poiché la natura dell'animo è da ritenersi mortale.

E come nel tempo passato non sentimmo alcun dolore

quando i Punici vennero da ogni parte all'assalto,

e tutto il mondo scosso dal trepido tumulto

tremò rabbrividendo sotto le alte volte dell'etere,

e fu in dubbio sotto il regno di quale fra i due popoli

dovessero cadere tutti gli uomini in terra e in mare,

così, quando non esisteremo più e si produrrà la separazione

del corpo e dell'anima, dalla cui congiunzione siamo formati,

di certo assolutamente nulla potrà accadere a noi,

che allora non più saremo, né stimolare i nostri sensi,

neanche se la terra si mischierà al mare e il mare al cielo.

Qui il poeta, tramite l'uso parodistico di un linguaggio di stampo enniano, costruisce una rappresentazione "gigantomachica" delle Guerre puniche. ${ }^{8}$ Lo scontro tra Romani e Cartaginesi coinvolge il mondo intero, sino ad abbracciare lo spazio marino e lo spazio celeste (vv. 835-837) e, come nello scontro tra Tifeo e Zeus nella Teogonia esiodea, la terra trema e il cosmo sembra sul punto di crollare (vv. 834-835): in palio vi è il dominio su tutti i popoli e sul mondo intero (v. 837).

Al di là dei riferimenti epici, è evidente in questo passo la polemica nei confronti di quella propaganda che, sin dal secondo secolo a.C., aveva celebrato Roma come padrona del mondo. Non sfugge infatti la scelta da parte del poeta del sostantivo regnum, termine sovente usato con accezione negativa per indicare un potere assoluto e dittatoriale, senza mettere in luce alcuna differenza politica tra Romani e Poeni. ${ }^{9}$ Ma soprattutto che valore ha il dominio sull'ecumene, se agli occhi di un Epicureo il mondo stesso non è che un punto in confronto all'universo (cfr. 6.649-652) e se la natura è capace in qualsiasi momento di condurre uomini, imperi e pianeti alla rovina (cfr. 5.105-106; 12261235)? Queste premesse aiutano a comprendere perché la descrizione del più importante evento della storia romana sia significativamente inserita in un contesto volto ad evidenziare l'inconsistenza del passato e la sua incapacità di condizionare il presente e il futuro. L'iperbolica rappresentazione dell'epico conflitto tra Roma e Cartagine, fondata su una sorta di crescendo apocalittico, si rivela infine un evento irrilevante. Questa ironica "deflazione del sublime escatologico" (procedimento che verrà esaminato nell'analisi del sesto libro lu-

8 Per un commento al passo, cfr. Luciani 2002.

9 Sull'ambigua accezione di regnum in età tardo-repubblicana cfr. Gisborne 2005, 118. Cfr. Lévy 2005, 62-66 in relazione alla posizione "antimilitarista" di Lucrezio. 
creziano $)^{10}$ è coronata dall'adynaton finale, il cui carattere apocalittico è paradossalmente volto a riaffermare l'ininfluenza sul presente delle catastrofi del passato e del futuro (vv. 840-842).

La descrizione della confusione tra i tre spazi in cui la tradizione poetica suddivideva il mondo (terra, mare e cielo) non rappresenta qui una mera figura retorica. ${ }^{11}$ L'adynaton diviene al contrario un potente veicolo della visione del mondo epicurea; esso non delinea un evento impossibile, un absurdum, bensì "svela" il reale destino del mondo: il ritorno alla caotica confusio elementorum. ${ }^{12}$ Tale rivelazione non è però inquietante perché conferma che anche la catastrofe più sconvolgente è incapace di turbare l'uomo e che la mortalità è in fondo garanzia di $\mu \alpha \kappa \alpha \rho$ เótns per il genere umano.

\subsection{Due “trionfi del tempo": le sezioni conclusive dei libri terzo e quarto}

Come un fiume carsico, il tema della fine del mondo sembra quasi scomparire (fatta eccezione per i casi sopra indicati) alla fine del secondo libro per riemergere soltanto al principio del quinto. Eppure vi è una sorta di filo rosso che rende meno traumatico questo "salto" di due libri e che rende il lettore conscio della continuità dell'argomentare lucreziano: si tratta del motivo della vetustas, ${ }^{13}$ che riemerge chiaramente nei finali di ciascuno di questi libri, con una forte caratterizzazione comico-diatribica. ${ }^{14}$ Come infatti si è visto, ${ }^{15}$ il primo libro si conclude con un trionfo del «tempo della conoscenza», ${ }^{16}$ che rende possibile la progressiva comprensione dell'intero universo da parte dell'individuo, laddove il

10 Cfr. pp. 203-206.

11 A proposito della fortuna del motivo dell'avvento della confusione, vera e propria formula proverbiale nell'età antica, cfr. Dutoit 1936 e Indelli 2014, 114-115.

12 Ritroviamo un uso analogo dell'adynaton nella tragedia senecana. Cfr. Mazzoli 1992, 137 «per l'adynaton si produce nelle tragedie di Seneca un drastico rovesciamento di segno. Lungi dal ridursi a mera risorsa formale, il suo uso si fa portatore di senso e ubbidisce a una profonda ragione ideologica».

13 Molto utile il raffronto con la riflessione del Varrone menippeo riguardo al medesimo tema. Si veda in proposito Leonardis 2014.

14 A proposito della comune caratterizzazione comico-diatribica di questi finali, più densi di riferimenti all'orizzonte romano contemporaneo al poeta rispetto alle altre sezioni, cfr. GellarGoad 2012, 282-286. Per un'analisi della filosofia e poetica del tempo nell'opera lucreziana, cfr. Luciani 2000

15 Cfr. pp. 68-70.

16 Cfr. Romano 2008. 
secondo si chiude con un "trionfo del tempo" tout court, rappresentato come la sola forza capace di condurre ogni cosa alla distruzione. ${ }^{17}$ In questo contesto, l'utilizzo del motivo della vetustas (si ricordi la perifrasi finale del v. 1174 spatio aetatis [...] vetusto) è funzionale alla dimostrazione della mortalità della terra e del mondo.

Il medesimo motivo riappare, con precisi richiami verbali e contenutistici al finale del secondo libro, anche nelle conclusioni del terzo e del quarto libro, creando una notevole sequenza di "trionfi del tempo" declinati diversamente a seconda dei contesti. ${ }^{18}$ Si consideri innanzi tutto il finale del terzo libro: compiuta la transizione dall'escatologia cosmica dei primi due libri all'escatologia individuale, il poeta costruisce un quadro dominato dalla "morte immortale ed eterna" (mors aeterna, vv. 1085-1094):

Posteraque in dubiost fortunam quam vehat aetas,

quidve ferat nobis casus quive exitus instet.

Nec prorsum vitam ducendo demimus hilum

tempore de mortis nec delibare valemus,

quominus esse diu possimus forte perempti.

Proinde licet quot vis vivendo condere saecla;

1090

mors aeterna tamen nilo minus illa manebit,

nec minus ille diu iam non erit, ex hodierno

lumine qui finem vitai fecit, et ille,

mensibus atque annis qui multis occidit ante.

1085 fortunam $A B$ : fortuna $O Q 1088$ delibare $A B$ : deliberare $O Q \quad 1089$ possimus $O^{1}$ : possumus $O Q$ forte] sorte ed. Aldina: morte Lambinus 1090 quot (vid. 317) : quod $O Q$ condere $O$ : ducere $Q$

È dubbio che cosa ci porti il tempo futuro, 1085 cosa ci rechi il caso, quale esito incalzi.

E certo protraendo la vita non sottraiamo un istante al tempo della morte, non riusciamo neanche a scalfirlo, per far sì che possiamo meno a lungo essere morti.

Ti è lecito dunque seppellire vivendo quante generazioni vuoi;

1090 tuttavia ti aspetterà non meno quell'eterna morte,

17 Cfr. Appendice 1.

18 Sull'importanza dei finali del De rerum natura (anche nella loro contrapposizione agli scenari proemiali), cfr. Minadeo 1969, 53: «after Book II, the surface dimensions narrow, drastically in III and IV, where Lucretius turns to analyze atomic processes that reside chiefly in the individual himself, and, meanwhile, the broadening of scope in the final two books never quite reattains the literal universal compass of I and II. Yet, as far as art can provide, the universal setting is never submerged». Tale aspetto è stato evidenziato anche da Klingner 1952, 139-144, che però imputa l'attenzione del poeta a questo tipo di scenari al suo "pessimismo". Cfr. la critica di Segal 1998, 118 n. 9. 
né meno a lungo non sarà esistente colui che termina oggi il corso della vita, di colui che da molti mesi e da molti anni è già prima scomparso.

A differenza dei due libri precedenti, volti all'esame dell'infinitamente piccolo o dell'immensamente grande, l'obbiettivo del poeta è ora focalizzato sulla sola realtà umana: il dominio della mors immortalis è infatti qui riferito in primis alla fine che attende ciascun individuo. Si noti come, analogamente al finale del secondo libro (cfr. 2.1150-1163), il poeta accresca il pathos tramite la scelta della prima persona plurale (v. 1086-1089) per poi passare a un altrettanto efficace seconda persona con valore impersonale (v. 1090). Altro elemento comune è l'insistenza sul campo semantico del tempo, nuovamente articolato con incredibile varietà (cfr. e.g. aetas; saecla; tempore; mortis; vita; hodierno lumine; mensibus; annis). Infine, come nel caso di 2.1173-1174, ${ }^{19}$ è presente una chiusura “epifonematica”, che attinge a un repertorio di modi di dire (si pensi al vecchio che «seppellisce molte generazioni» al v. 1090) elevati a un registro tragico, che sfocia nel sublime v. 1091.

Anche nel finale del quarto libro, dedicato, com'è noto, alla tematica amorosa, Lucrezio decide di collocare una chiusa sentenziosa, nuovamente strutturata come un trionfo del tempo (4.1283-1287): ${ }^{20}$

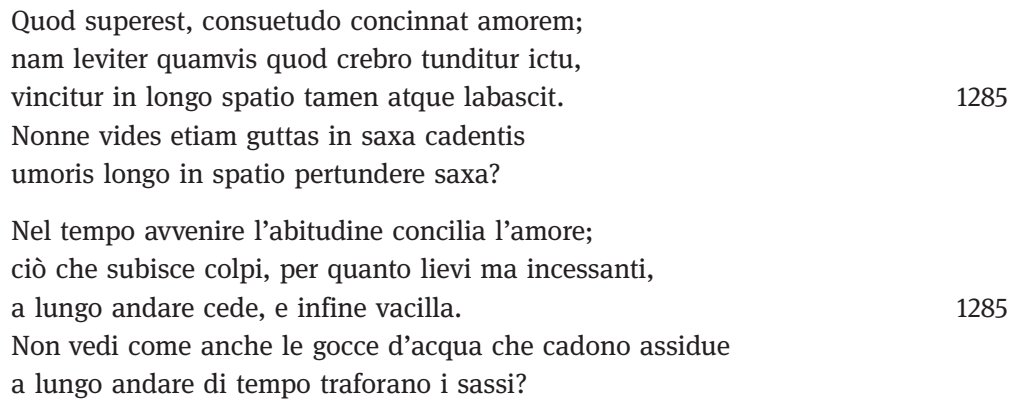

La tematica erotica diviene nuovamente occasione per ribadire il potere della vetustas, con il ritorno del motivo dello spazio di tempo (v. 1285 in longo spatio; v. 1287 longo in spatio) capace di logorare e vincere ogni aggregato. In questo modo, anche la consuetudine si trasforma in una sorta di assedio atomico proveniente dallo spazio esterno, che alla fine piega ogni avversario in virtù della pressione costante (v. 1284 crebro tunditur ictu); in questo gioco di corri-

19 Cfr. Appendice 1. A proposito dei vv. 1090-1091, cfr. Usener 1913, 118-119.

20 Per un'analisi di questo finale cfr. il commento di Minadeo 1969, 36 e Brown 1987, 377-380. 
spondenze con i finali precedenti, anche il tabescere di 2.1173 trova un parallelo nel labascere di 4.1285. E ancora una volta il poeta ricorre a una chiusa squisitamente diatribica, con il ricorso alla seconda persona con valore impersonale in una interrogativa retorica (nonne vides) chiusa da un motivo proverbiale (stavolta il noto gutta cavat lapidem).

Questa rapida rassegna dimostra che i finali lucreziani dei libri centrali del poema (con l'esclusione del quinto) si configurano come trionfi dei moti atomici disgregatori (motus exitiales, 2.569) destinati a dissolvere tutti gli aggregati, dagli esseri viventi al mondo. In particolare, il finale del terzo libro rappresenta un passo cruciale nel cammino del lettore lucreziano, anticipando la sua tappa finale, ossia la conclusione dell'intero poema: nella potente allegoria dell'episodio della Peste di Atene nel sesto libro, infatti, la ruina della dimensione civica diverrà perfetta sintesi tra la ruina individuale cantata nel finale del terzo e la ruina cosmica prospettata al termine dei primi due libri. L'analisi congiunta dei finali del poema lucreziano permette inoltre di comprendere come tra l'uomo, il mondo e ogni aggregato atomico nell'universo vi sia una relazione di continuità: la differenza tra essi è solamente quantitativa, e non qualitativa. ${ }^{21}$ Se alla base della teologia astrale di ascendenza platonica vi era la sacra correlazione tra l'eternità spirituale dell'individuo e l'eternità del mondo, Lucrezio, negando l'esistenza di un'anima immortale (individuale e cosmica), "rovescia" tale correlazione, evidenziando come uomo e mondo siano parimenti vulnerabili e soggetti alla vetustas. ${ }^{22}$ In questo modo, la dimostrazione della mortalità dell'uno permette di consolidare la dimostrazione della mortalità dell'altro. Non è un caso che nel cuore dell'escatologia cosmica lucreziana, ovverosia i vv. 91-415 del quinto libro, il poeta ricorra - come si vedrà - alla trasposizione di argomentazioni già elaborate nel terzo libro a proposito della mortalità individuale.

21 Cfr. Segal 1998, 118-119, che sottolinea come morte individuale, civica e cosmica siano per Lucrezio poste sul medesimo piano: «il microcosmo e il macrocosmo non sono solo analoghi tra di loro, ma sono parte dello stesso continuum. Dal punto di vista psicologico, tuttavia, le analogie tra la morte del mondo e la morte della persona servono a preparare il lettore ad affrontare nel terzo libro la realtà della sua morte individuale. Per questa ragione, forse, Lucrezio anticipa gli argomenti del quinto libro e sceglie di terminare i primi due libri con intensi racconti di cataclismi».

22 Cfr. pp. 126-130. 